\title{
ĐỊNH VỊ ĐIỂM CHÍNH XÁC Vớl CÁC MÁY THU GPS RẺ TIỀN
}

\author{
TS. NGUYẼ̂N NGỌC LÂU \\ Trường Đại học Bách khoa TP. HCM
}

\section{Tóm tắt:}

Chúng tôi đã tiến hành thu thập dữ liệu của một số máy thu GPS cầm tay Garmin và xử lý tuyệt đối bằng 2 phương pháp i) hiệu chỉnh độ trễ điện ly bằng mô hình IGS và ii) khảo sát các tham số độ trễ điện ly thiên đỉnh và gradient. Kết quả xử lý cho thấy độ chính xác định vị tăng theo thời gian thu. Với thời gian thu từ 5 phút đến hơn 300 phút, độ chính xác thành phẩn mặt bằng là $1.50-0.15 \mathrm{~m}$ và thành phần độ cao là $2.70-0.60 \mathrm{~m}$. Phương pháp ii) có thể cải thiện độ chính xác tốt hơn so với phương pháp i) khoảng $0.2 m$ và $0.8 m$ tương ứng với mặt bằng và độ cao.

\section{Giới thiệu}

inh vị điểm chính xác cao thường sử dụng các máy thu GPS hai tần số và khai thác các sản phẩm chính xác của tổ chức IGS như bản lịch vệ tinh và số hiệu chỉnh đồng hồ vệ tinh. Độ chính xác đạt được khoảng $5 \mathrm{~mm}$ cho mă̆t bằng và $1 \mathrm{~cm}$ cho độ cao khi đo tĩnh 24 giờ [11]. Các máy thu một tần số ít được chú ý trong định vị điểm chính xác cao vì khả năng loại trử ảnh hưởng tầng điện ly kém, không hiệu quả. Đặc biệt các máy thu GPS cầm tay chỉ cung cấp tọa độ với độ chính xác 5-15 m.

Có nhiều nghiên cứu về định vị điểm chính xác với máy thu GPS một tần số, tuy nhiên tập trung vào các máy thu GPS rẻ tiền thì Beran $[7,9,14]$ là người đầu tiên. Tác giả đã đề xuất dùng bộ lọc Kalman để xử lý trị đo mã và trị đo pha ở dạng hiệu thời gian. Các trị đo này đã được hiệu chỉnh độ trễ điện ly từ lưới ô vuông của IGS. Một số kết quả định vị tĩnh được cho ở bảng 1 :

Bảng 1: Kết quả định vị điểm của Beran

\begin{tabular}{|c|c|c|c|c|c|}
\hline $\begin{array}{l}\text { Ch. dài } \\
\text { dữ liệu } \\
\text { (giờ) }\end{array}$ & $\begin{array}{l}\text { Thành } \\
\text { phần }\end{array}$ & $\begin{array}{l}\text { TB } \\
(\mathrm{m})\end{array}$ & $\begin{array}{l}\text { Std } \\
(\mathrm{m})\end{array}$ & $\begin{array}{l}\text { SSTP } \\
(\mathrm{m})\end{array}$ & Ghi chú \\
\hline 1.0 & $\begin{array}{c}\text { North } \\
\text { East } \\
\text { Up }\end{array}$ & & $\begin{array}{l}1.27 \\
0.36 \\
0.97\end{array}$ & $\begin{array}{l}2.70 \\
1.88 \\
4.99 \\
\end{array}$ & $\begin{array}{c}\text { Máy } 12 \mathrm{XL} \\
\text { theo tài liệu [5] }\end{array}$ \\
\hline 3.5 & $\begin{array}{c}\text { North } \\
\text { East } \\
\text { Up }\end{array}$ & $\begin{array}{l}+0.47 \\
-0.54 \\
-0.69 \\
\end{array}$ & $\begin{array}{l}0.39 \\
0.14 \\
0.85\end{array}$ & $\begin{array}{l}0.61 \\
0.56 \\
1.10 \\
\end{array}$ & $\begin{array}{c}\text { Máy } 12 \mathrm{XL} \\
\text { theo tài liệu [7] }\end{array}$ \\
\hline
\end{tabular}

Satirapod và Kriengkraiwasin [10] đã sử dụng bộ lọc Kalman mở rộng xử lý kết hợp các trị đo $L 1$ và $P 1$ từ các máy thu GPS 2 tần số tại 5 trạm IGS có tốc độ thu 30 giây. Kết quả cho thấy độ xác định vị mặt bằng phụ thuộc vào thời gian đo theo bảng sau:

Bảng 2: Độ chính xác định vị mặt bằng theo thời gian đo [10]

\begin{tabular}{|c|l|}
\hline Chiều dài ca đo (phút) & SSTP $(\mathrm{m})$ \\
\hline 5 & 2.44 \\
\hline 10 & 2.04 \\
\hline 15 & 1.94 \\
\hline 30 & 1.51 \\
\hline
\end{tabular}




\section{Deghiên cuíre - Cíng dụng}

Trong tài liệu [13], chúng tôi đã giới thiệu phần mềm RINEXLogger do chúng tôi tự viết. Phần mềm có khả năng thu được trị đo giả cự ly $C / A$ và trị đo pha trên tần số $L 1$ từ các máy thu GPS cầm tay của hãng Garmin. Đặc thù của các trị đo này là có độ nhiễu cao do sử dụng anten đơn giản và trượt chu kỳ pha xảy ra nhiều hơn các máy thu trắc địa khoảng 3 lần [14]. Sai số đồng hồ máy thu trong các trị đo biến động rất lớn. Trong ba giờ thu dữ liệu, đồng hồ máy thu có thể sai 0.2-0.3 giây.

Trong bài báo này, chúng tôi muốn khảo sát độ chính xác định vị điểm của những máy thu GPS cầm tay nêu trên bằng hai phương pháp: i) áp dụng số hiệu chỉnh tầng điện ly từ tổ chức IGS và ii) xem nó là một ẩn số để xử lý trong mô hình. Chúng tôi cũng áp dụng bộ lọc Kalman để xử lý nhưng sử dụng trị đo hiệu đơn giữa các vệ tinh.

\section{Mô hình xử lý định vị điểm}

Các vệ tinh GPS truyền tín hiệu trên 2 băng sóng tải $L 1$ và L2 với tần số $1575.42 \mathrm{MHz}$ và $1227.60 \mathrm{MHz}$. Tuy nhiên các máy thu một tần số chỉ cung cấp được trị đo giả cự ly mã C/A và trị đo pha tích hợp trên tần số $L 1$. Các trị đo này được mô hình như sau $[3,4,8]$ :

$$
\begin{gathered}
\left.\Phi_{i, 1}^{k}(t)=\rho_{i}^{k}(t)-I_{i, 1}^{k}+T_{i}^{k}+\delta m_{i, 1}^{k}+c \mid d t_{i}(t)-d t^{k}\left(t-\tau_{i}^{k}\right)\right\rfloor+\lambda_{1} N_{i, 1}^{k}+\varepsilon_{i, 1}^{k} \\
C_{i, 1}^{k}(t)=\rho_{i}^{k}(t)+I_{i, 1}^{k}+T_{i}^{k}+d m_{i, 1}^{k}+c\left\lfloor d t_{i}(t)-d t^{k}\left(t-\tau_{i}^{k}\right)\right\rfloor+e_{i, 1}^{k}
\end{gathered}
$$

Trong đó:

* $\Phi_{i, 1}^{k}$ là trị đo pha trên tần số $L 1$ giữa máy thu i và vệ tinh $\mathrm{k}$ ở thời điểm thu $\mathrm{t}$ thu $\mathrm{t}$

* $C_{i, 1}^{k}(t)$ là trị đo giả cự ly mã $C / A$ trên tần số L1 giữa máy thu i và vệ tinh $\mathrm{k}$ ở thời điểm

* $\rho_{i}^{k}(t)$ là khoảng cách hình học giữa máy thu i và vệ tinh $\mathrm{k}$ ở thời điểm thu $\mathrm{t}$

* $I_{i, 1}^{k}$ là độ trễ điện ly trên tần số $L 1$

* $T_{i}^{k}$ là độ trễ đối lưu theo đường truyền tín hiệu

* $\delta m_{i, 1}^{k}$ là ảnh hưởng của đa đường trên trị đo pha tần số L1

* $d m_{i, 1}^{k}$ là ảnh hưởng của đa đường trên trị đo giả cự ly mã C/A

* $\mathrm{c}=299792.458 \mathrm{~km} / \mathrm{s}$ là vận tốc của ánh sáng trong chân không

* $d t_{i}(t)$ là sai số đồng hồ máy thu ở thời điểm thu $\mathrm{t}$

* $d t^{k}\left(t-\tau_{i}^{k}\right)$ là sai số đồng hồ vệ tinh ở thời điểm phát

* $N_{i, 1}^{k}$ và là tham số đa trị trên tần số $\mathrm{L} 1$

* $\lambda_{1}$ là bước sóng của tần số $L 1 ; \lambda_{1}=\frac{c}{f_{L_{1}}}$

* $\varepsilon_{i, 1}^{k}$ là sai số đo của trị đo pha trên tần số L1

* $e_{i, 1}^{k}$ là sai số đo của trị đo giả cự ly trên tần số L1

2.1. Mô hinh xử lý của Beran

Beran $[7,9,14]$ đã đề xuất dùng trị đo pha ở dạng hiệu thời gian tương tự như trị đo độ lệch tần số Doppler [3] như sau:

$$
\nabla \Phi_{i, 1}^{k}(t)=\nabla \rho_{i}^{k}(t)-\nabla I_{i, 1}^{k}+\nabla T_{i}^{k}+\nabla \delta m_{i, 1}^{k}+c\left[\nabla d t_{i}(t)-\nabla d t^{k}\left(t-\tau_{i}^{k}\right)\right]+\nabla \varepsilon_{i, 1}^{k}
$$


Trong đó: $\nabla$ là toán tử hiệu giữa hai epoch liên tiếp, ví dụ $\nabla \Phi_{i, 1}^{k}(t)=\Phi_{i, 1}^{k}(t+\Delta)-\Phi_{i, 1}^{k}(t)$

Có thể thấy ưu điểm của việc sử dụng phương trình (3) là không có mặt tham số đa trị. Trị đo (3) chịu ảnh hưởng của hiện tượng trượt chu kỳ pha là ít nhất. Hơn nữa các ảnh hưởng của tâng điện ly và đối lưu ở hai epoch liên tiếp rất tương tự như nhau nên hiệu của chúng có giá trị rất nhỏ.

Mối liên hệ giữa các trị đo khoảng cách của cùng một vệ tinh GPS thu được ở hai thời điểm khác nhau đã được đề cập trong nhiều tài liệu $[3,4,8]$. Mối liên hệ này càng cao khi hai thời điểm đo càng gần nhau. Để đơn giản hóa quá trình xử lý, người ta thường giảm tốc độ thu để giảm sự liên hệ và bỏ qua nó. Việc thành lập các trị đo kiểu (3) làm cho mối liên hệ giữa chúng càng tăng lên. Điều này sẽ không là vấn đề nếu sử dụng mô hình sai số tương đương. Tuy nhiên mô hình sai số này sẽ làm cho quá trình xử lý cực kỳ phức tạp và người ta lại phải giả sử các trị đo (3) là không liên hệ với nhau.

\subsection{Mô hình xử lý của Satirapod và Kriengkraiwasin}

Satirapod và Kriengkraiwasin [10] đã sử dụng kết hợp các trị đo pha và mã tương tự như Witchayangkoon [5] và Gao and Shen [6]:

$$
0.5\left(\Phi_{i, 1}^{k}(t)+C_{i, 1}^{k}(t)\right)=\rho_{i}^{k}(t)+T_{i}^{k}+0.5\left(\delta m_{i, 1}^{k}+d m_{i, 1}^{k}\right)+c\left\lfloor d t_{i}(t)-d t^{k}\left(t-\tau_{i}^{k}\right)\right\rfloor+0.5 \lambda_{1} N_{i, 1}^{k}+0.5\left(\varepsilon_{i, 1}^{k}+e_{i, 1}^{k}\right)
$$

Phương trình (4) cho thấy đã khử hoàn toàn ảnh hưởng của tầng điện ly. Do đó không cần phải hiệu chỉnh hay khảo sát tham số đó trong mô hình. Nhược điểm của (4) là chịu ảnh hưởng đa đường và độ nhiễu cao từ trị đo mã. Nếu dùng trị đo mã $\mathrm{C} / \mathrm{A}$ thì độ nhiễu cao hơn mã $\mathrm{P}$ khoảng 10 lần.

\subsection{Mô hình của chúng tôi}

Nếu ta sử dụng các sản phẩm chính xác của tổ chức IGS về tọa độ vệ tinh $(\sim 5 \mathrm{~cm})$ và sai số đồng hồ vệ tinh $(\sim 0.1 \mathrm{~ns})$, thì số hạng $d t^{k}\left(t-\tau_{i}^{k}\right)$ trong phương trình (1) và (2) có thể bỏ qua.

Đối với ảnh hưởng của tầng đối lưu, ta có thể dùng các mô hình có sẳn của Saastamoinent [1] để tính độ trễ đối lưu thiên đỉnh theo các tham số khí tượng với độ chính xác vài $\mathrm{cm}$. Sau đó dùng hàm ánh xạ Niell [2], để chuyển thành độ trễ đối lưu theo hướng bất kỳ:

$$
T_{i}^{k}=m\left(z_{i}^{k}\right) T_{i}^{Z}
$$

trong đó $m(z)$ là hàm ánh xạ Niell, $z$ là góc thiên đỉnh của hướng truyền tín hiệu và Tª là độ trễ đối lưu thiên đỉnh.

Sai số đồng hồ máy thu có thể được xem là một ẩn số để xử lý. Tuy nhiên do các máy thu GPS cầm tay thường trang bị chuẩn đồng hồ thạch anh có độ ổn định không cao nên rất khó mô hình hóa sự biến động của chúng. Cách thức hiệu quả nhất là dùng phương trình hiệu giữa hai vệ tinh khác nhau (ví dụ là vệ tinh $\mathrm{k}$ và $\mathrm{l}$ ) để khử hoàn toàn sai số này:

$$
\begin{gathered}
\Delta \Phi_{i, 1}^{k l}(t)=\Delta \rho_{i}^{k l}(t)-\Delta V_{i, 1}^{k l}+\lambda_{1} \Delta N_{i, 1}^{k l}+\Delta \varepsilon_{i, 1}^{k l} \\
\Delta C_{i, 1}^{k}(t)=\Delta \rho_{i}^{k l}(t)+\Delta V_{i, 1}^{k l}+\Delta e_{i, 1}^{k}
\end{gathered}
$$

Chúng tôi xử lý đồng thời cả hai trị đo pha (6) và trị đo mã (7) với trọng số thích hợp.

\section{Khắc phục ảnh hưởng tầng điện ly}


Trong phương trình (4) và (5) vẫn còn xuất hiện ảnh hưởng của tầng điện ly I. Để phân tích số hạng này người ta thường mô hình hóa thành

$$
I_{i, 1}^{k}=m_{I}\left(z_{i}^{k}\right) I_{i, 1}^{z}
$$

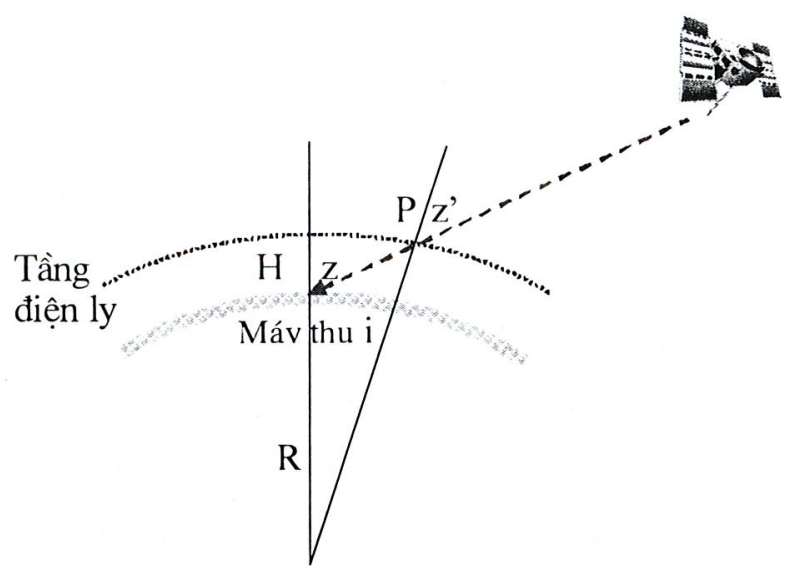

Hình 1: Hàm ánh xạ lớp đơn của tầng điện ly

Trong đó:

* $I_{i, 1}^{z}$ là độ trễ điện ly thiên đỉnh tại máy thu i

* $m_{1}$ là hàm ánh xạ lớp đơn của tầng điện ly được tính theo công thức (xem hình 1)

$$
m_{l}=\frac{1}{\cos z^{\prime}} \text { với } \sin z^{\prime}=\frac{R}{R+H} \sin z
$$

* $Z$ và $z$ ' là góc thiên đỉnh của hướng truyền tín hiện tại vị trí máy thu và tại điểm $P ; R$ là bán kính trung bình của trái đất (thường chọn là $6371 \mathrm{~km}$ ) và $\mathrm{H}$ là độ cao tầng điện ly so với mặt đất (thường chọn là $400 \mathrm{~km}$ ).

Trong bài báo này, chúng tôi xử lý mô hình (8) theo hai phương pháp:

\subsection{Dùng lưới ô vuông TEC từ tổ chức IGS}

TEC (Total Electron Content) là mật độ electron tích phân dọc theo đường truyền tín hiệu. TEC là một tham số thời tiết quan trọng đặc trưng cho trạng thái và động học của plasma trong tầng điện ly. Hiện nay tổ chức IGS đang cung cấp giá trị VTEC (TEC ở hướng thiên đỉnh) trên một lưới ô vuông kinh, vĩ độ $5 \times 2.5$ độ với độ chính xác tốt nhất là 2-8 TECU (tương đương $0.32-1.28 \mathrm{~m}$ ) (xem bảng 3 ). Giá trị trong lưới được cho mỗi 2 giờ. Khi cần tính toán VTEC tại một vị trí bất kỳ ở một thời điểm bất kỳ ta cần phải nội suy từ lưới ô vuông này.

Bảng 3: Độ chính xác sản phẩm TEC của IGS

\begin{tabular}{|l|l|l|l|}
\hline Sản phẩm IGS & $\begin{array}{l}\text { Độ chính } \\
\text { xác }\end{array}$ & $\begin{array}{l}\text { Thời gian } \\
\text { nhận SP }\end{array}$ & Lưới ô vuông \\
\hline Final ionospheric TEC grid & $2-8$ TECU & $\sim 11$ ngày & $\begin{array}{l}2 \text { giờ, } \\
5 \text { deg (lon) x 2.5 deg (lat) }\end{array}$ \\
\hline Rapid ionospheric TEC grid & $2-9$ TECU & $<24$ giờ & $\begin{array}{l}2 \text { giờ; } \\
5 \text { deg (lon) x 2.5 deg (lat) }\end{array}$
\end{tabular}


Độ trễ đối lưu thiên đỉnh $\mathrm{I}^{2}$ có thể tính từ VTEC theo công thức sau:

$$
I^{Z}=\frac{40.3 \times V T E C}{f^{2}} 10^{16}
$$

Trong đó $\mathrm{f}$ là tần số của sóng tải GPS ( $\mathrm{f}=1575.42 \mathrm{~Hz}$ ).

3.2. Coi độ trễ điện ly thiên đỉnh $I_{i, 1}^{z}$ là ẩn số

Chúng ta có thể coi các tham số $I_{i, 1}^{z}$ là ẩn số bổ sung để xử lý cùng với các tham số tọa độ máy thu. Tuy nhiên:

$I_{i, 1}^{z}$ biến động theo thời gian, nên chúng tôi dùng bộ lọc Kalman để xử lý và mô hình các tham số điện ly là bước ngẫu nhiên (random walk) với tần số cập nhật là 15 phút/lần.

$I_{i, 1}^{z}$ biến động theo vị trí của điểm P. Nếu chỉ dùng một tham số duy nhất $I_{i, 1}^{z}$ sẽ không cho độ chính xác mong muốn vì ảnh hưởng điện ly còn thể hiện ở các thành phẩn gradient trên mặt phẳng. Do đó trong xử lý, chúng tôi khảo sát ba tham số liên quan đến độ trễ điện ly gồm $a_{0}, a_{1}$ và $a_{2}$ theo mô hình tương tự như mô hình độ trễ ở tầng đối lưu [8]:

$$
I_{i, 1}^{z}=a_{0}+\tan z_{i}^{\prime k}\left(\sin \alpha_{i}^{k} \cdot a_{1}+\cos \alpha_{i}^{k} \cdot a_{2}\right)
$$

trong đó $\alpha_{1}^{k}$ là góc phương vị vệ tinh $\mathrm{k}$; $\mathrm{a}_{0}$ đóng vai trò là độ trễ điện ly thiên đỉnh tại điểm $\mathrm{i} ; \mathrm{a}_{1}$ và $\mathrm{a}_{2}$ là thành phần gradient hướng Đông và hướng Bắc của điểm $\mathrm{P}$ so với điểm $\mathrm{i}$.

\section{Thu thập và xử lý dữ liệu}

Chúng tôi sử dụng một số máy thu Garmin khác nhau đặt trên cùng một điểm trên mặt đất. Tọa độ điểm này đã được xác định bằng máy thu GPS hai tần số với độ chính xác $\mathrm{cm}$. Dữ liẹu đo từ các máy thu này được lấy ra bằng phần mềm RINEXLogger [13]. Bảng 4 tóm tắt loại máy thu GPS cầm tay Garmin được sử dụng trong khảo sát.

Bảng 4: Các máy thu GPS Garmin dùng trong khảo sát

\begin{tabular}{|l|c|c|c|}
\hline Máy thu & Ngày của 2009 & Tốc độ thu (s) & Thời gian thu $(\mathrm{h})$ \\
\hline III+ & 243 & 5 & 5.3 \\
12XL & 252 & 5 & 4.0 \\
GPSmap 60 & 271 & 5 & 3.2 \\
GPSmap 76CS & 274 & 5 & 3.0 \\
GPSmap 76CS & 280 & 5 & 1.5 \\
\hline
\end{tabular}

Như đã dự báo trước, dữ liệu pha thu thập được tồn tại rất nhiều trượt chu kỳ. Nếu không được sửa chữa hiệu quả, nó chắc chắn sẽ ảnh hưởng đến kết quả xử lỳ. Chúng tôi áp dụng thuật toán đã đề xuất trong tài liệu [12] để sửa chữa các độ trượt. Nhưng vì dữ liệu pha ở đây chỉ có trên một tần số nên phải có sự cải tiến để thuật toán có thể hoạt động tốt. Hình 2 minh họa quá trình sửa chữa trị đo pha của một cặp vệ tinh $4-15$ của máy thu Garmin $12 X L$ ngày 243.

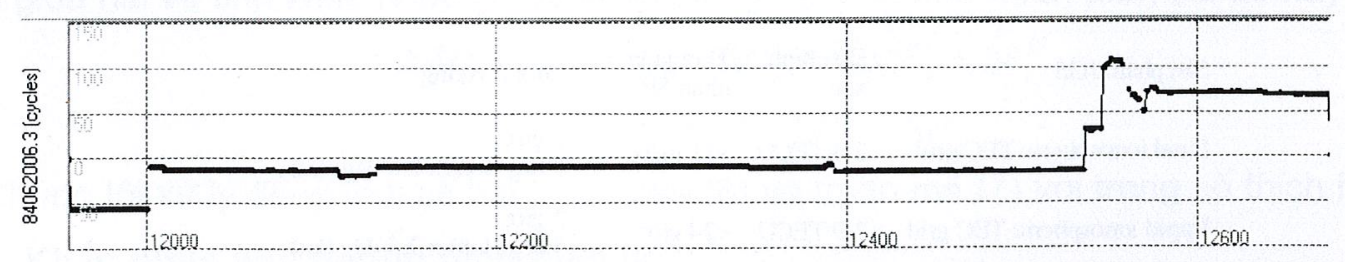




\section{Mghiên cuíu - Míng dụng}

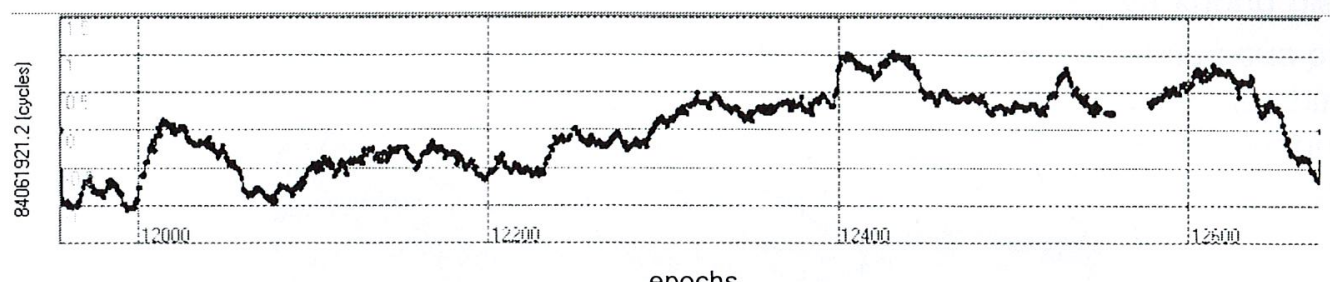

epochs

Hình 2: Trị đo pha L1 trước (cửa sổ trên) và sau (cửa sổ dưới) sửa chữa trượt chu kỳ

Để khảo sát độ chính xác định vị theo thời gian đo, chúng tôi lần lượt cắt dữ liệu thành các phân đoạn $5,15,30,60,90,120,180,240$ và 318 phút. Sau khi xử lý dữ liệu bằng hai phương pháp nêu trên, rhínn tôi nhân đi đơn kốt nıı̉ rhn ở hình vã 3 đốn 7 và bảng 4 .

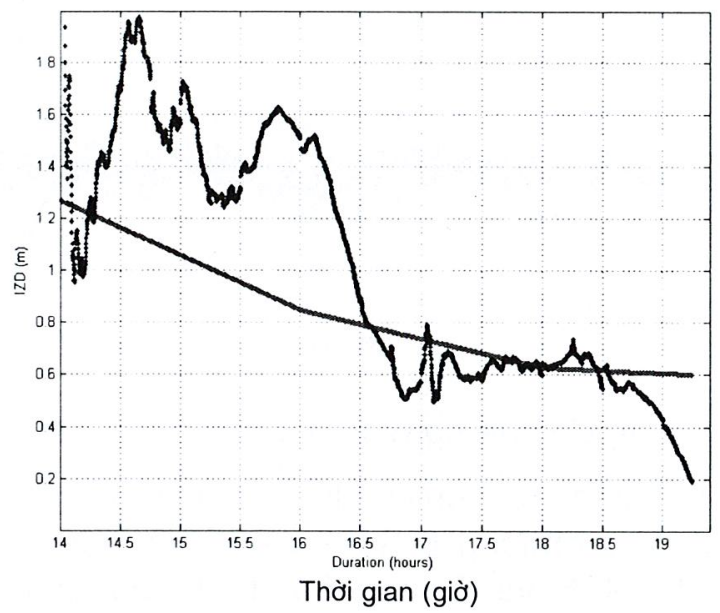

Hinh 3: Độ trễ điện ly thiên đỉnh ngày 243 theo phương pháp 1 (đỏ) và theo phương pháp 2 (xanh)

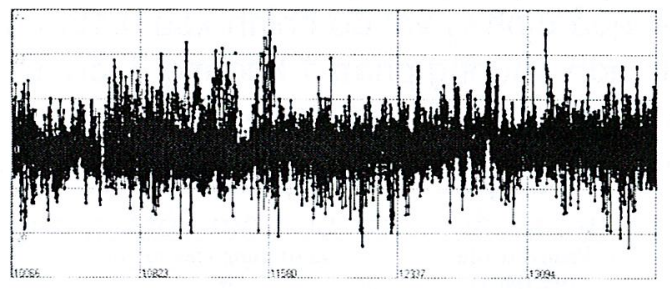

epochs

Hình 4: Phần dư trị đo giả cựly C1 ngày 243, phân đoạn 318 phút, phương pháp 1

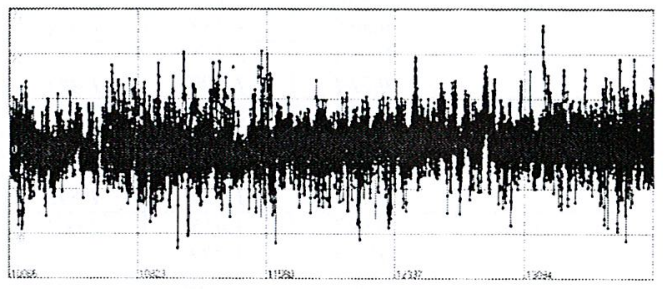

epochs

Hinh 5: Phần dư trị đo giả cựly C1 ngày 243, phân đoạn 318 phút, phương pháp 2 


\section{Mghiên cuíu - Míng dụng}

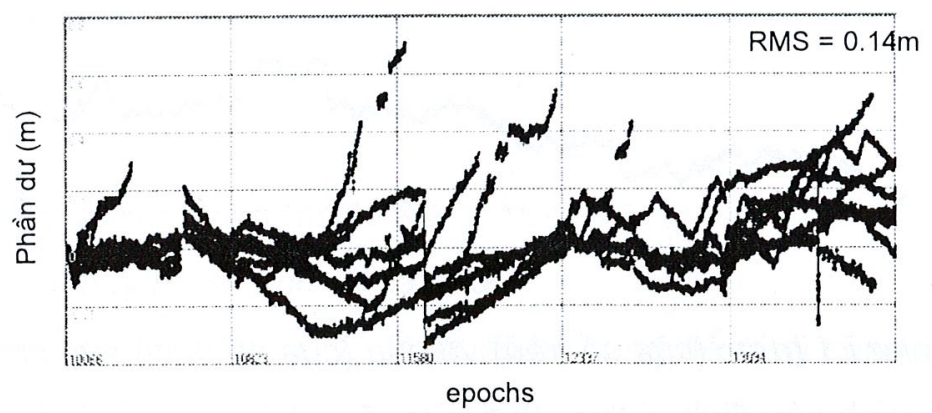

Hình 6: Phần dư trị đo pha ngày 243, phân đoạn 318 phút, phương pháp 1

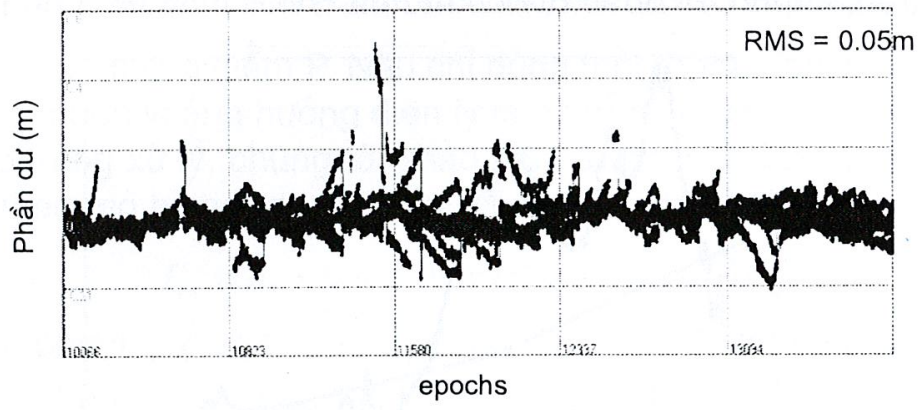

Hìn 7: Phần dư trị đo pha ngày 243, phân đoạn 318 phút, phương pháp 2

Các hình vẽ 4 và 5 thể hiện phần dư của trị đo mã khi xử lý dữ liệu máy thu III+ với phân đoạn dữ liệu 318 phút theo phương pháp 1 và 2 , cho sai số trung phương phần dư là $4.53 \mathrm{~m} / 4.39 \mathrm{~m}$. Các hình vẽ 6 và 7 là phần dư trị đo pha với sai số trung phương $0.14 \mathrm{~m} / 0.05 \mathrm{~m}$. Điều này cho thấy việc khảo sát tham số độ trễ điện ly cho sự cải thiện khoảng 3 lần trên các phần dư trị đo pha so với khi dùng số hiệu chỉnh IGS.

Phương pháp 2 cho kết quả định vị với độ chính xác $0.15 \mathrm{~m}$ ở thành phần mặt bằng và $0.54 \mathrm{~m}$ ở độ cao, chính xác hơn phương pháp 1 khoảng 2 lần. Điều này có thể do sử dụng độ trễ điện ly thiên đỉnh khác nhau, chênh lệch có lúc đến $0.7 \mathrm{~m}$ (xem hinh 3).

Bảng 5: Tổng hợp kết quả xử lý

\begin{tabular}{|c|c|c|c|}
\hline \multirow{2}{*}{$\begin{array}{l}\text { Phân đoạn dữ } \\
\text { liệu (phút) }\end{array}$} & \multicolumn{3}{|c|}{ Sai số trung phương (m) } \\
\hline & $m_{H}$ & $m_{U}$ & $m_{p}$ \\
\hline \multirow[t]{2}{*}{5} & 1.372 & 1.426 & 1.979 \\
\hline & 1.156 & 1.407 & 1.821 \\
\hline \multirow[t]{2}{*}{15} & 1.038 & 1.824 & 2.099 \\
\hline & 0.928 & 1.358 & 1.645 \\
\hline \multirow[t]{2}{*}{ 30) } & 0.938 & 2.128 & 2.326 \\
\hline & 0.865 & 1.252 & 1.522 \\
\hline \multirow[t]{2}{*}{60} & 0.988 & 2.211 & 2.422 \\
\hline & 0.714 & 1.300 & 1.483 \\
\hline \multirow[t]{2}{*}{90} & 0.914 & 2.492 & 2.654 \\
\hline & 0.576 & 1.343 & 1.461 \\
\hline \multirow[t]{2}{*}{120} & 0.828 & 2.669 & 2.794 \\
\hline & 0.427 & 1.479 & 1.539 \\
\hline \multirow[t]{2}{*}{180} & 0.587 & 1.915 & 2.003 \\
\hline & 0.224 & 1.034 & 1.058 \\
\hline \multirow[t]{2}{*}{240} & 0.395 & 1.504 & 1.555 \\
\hline & 0.199 & 0.672 & 0.701 \\
\hline \multirow[t]{2}{*}{318} & 0.280 & 1.359 & 1.388 \\
\hline & 0.149 & 0.539 & 0.559 \\
\hline
\end{tabular}


Bảng 5 thể hiện sai số trung phương vị tri mặt bằng $\mathrm{m}_{\mathrm{H}}$, độ cao $\mathrm{m}_{\cup}$ và không gian $\mathrm{m}_{\mathrm{p}}$. Các số in thường là kết quả của cách xử lý 1 . Các số in đậm, nghiêng là kết quả của phương pháp xử lý 2 . So sánh hai phương pháp ta thấy phương pháp 2 luôn luôn cho độ chính xác tốt hơn phương pháp 1 khoảng $0.2 \mathrm{~m}$ trên thành phần mặt bằng và $0.8 \mathrm{~m}$ trên thành phần độ cao. Trong mỗi phương pháp, độ chính xác độ cao luôn tệ hơn độ chính xác mặt bằng từ $1.2-4.8$ lần.

Cũng theo bảng 5 , cùng thời gian đo tương ứng, kết quả của Beran (bảng 1) và Satirapod (bảng 2) luôn cho độ chính xác tệ hơn kết quả của chúng tôi ở phương pháp 1. Điều này cho thấy dùng trị đo hiệu đơn giữa các vệ tinh sau khi sửa chữa trượt chu kỳ một cách hiệu quả có những ưu điểm nhất định so với khi dùng trị đo ở dạng hiệu thời gian [7, $9,14]$ hay kết hợp giữa trị đo mã và pha chất lượng cao [10].

\section{Kết luận}

Chúng tôi đã phân tích một số mô hình định vị điểm cho các máy thu một tần số. Trong bài báo này chúng tôi xử lý đồng thời trị đo pha và mã ở dạng hiệu đơn giữa các vệ tinh.

Từ thu thập dữ liệu thu thập được của một số máy thu GPS cầm tay Garmin và xử lý tuyệt đối bằng 2 phương pháp i) hiệu chỉnh độ trễ điện ly bằng mô hình IGS và ii) khảo sát các tham số độ trễ điện ly thiên đỉnh và gradient. Kết quả xử lý cho thấy:

* Độ chính xác định vị tăng theo chiều dài phân đoạn dữ liệu xử lý

* Độ chính xác độ cao tệ hơn độ chính xác mặt bằng 1.2 - 4.8 lần

* Phương pháp 2 luôn cho kết quả tốt hơn phương pháp 1 khoảng $0.2 \mathrm{~m}$ trên thành phần mặt bằng và $0.8 \mathrm{~m}$ trên thành phần độ cao.

* Dùng trị đo hiệu đơn giữa các vệ tinh sau khi sửa chữa trượt chu kỳ một cách hiệu quả có những ưu điểm nhất định so với khi dùng trị đo ở dạng hiệu thời gian hay kết hợp giữa trị đo mã và pha chất lượng cao.

* Kết quả của Beran và Satirapod có độ chính xác tồi hơn kết quả của chúng tôi ở phương pháp 1. Điều này cho thấy mô hình định vị điểm dùng trị đo hiệu đơn giữa các vệ tinh sau khi sửa chữa trượt chu kỳ một cách hiệu quả có những ưu điểm nhất định so với khi dùng trị đo ở dạng hiệu thời gian hay kết hợp giữa trị đo mã và pha chất lượng cao.

Hình 8: Mô hình hóa đô chính xác măt bằna

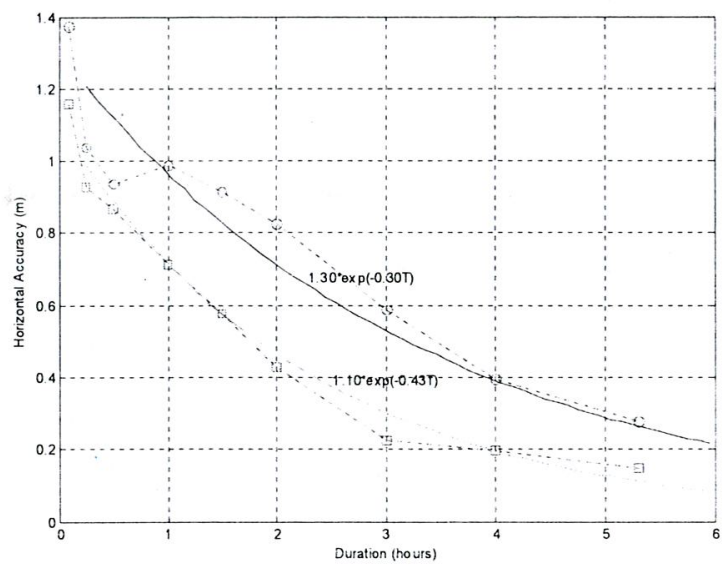




\section{Mghiên cuiue - Uíng dụng}

Nếu quan tâm đến thành phần mặt bằng, ta thấy qui luật biến thiên của độ chính xác theo thời gian gần với qui luật hàm mũ (xem hinh 8). Do đó có thể mô hình hóa sai số mặt bằng bằng công thức $\sigma_{H}=1.30 \times e^{-0.30 T}$ cho phương pháp 1 và $\sigma_{H}=1.10 \times e^{-0.43 T}$ cho phương pháp 2. Trong đó T là thời gian đo theo đơn vị giờ.

Dựa vào qui luật trên ta có thể kết luận đối với máy thu GPS cầm tay:

* Để đạt độ chính xác mặt bằng $1.0 \mathrm{~m}$, ta cần thu dữ liệu từ 15 phút trở lên

* Để đạt độ chính xác mặt bằng $0.5 \mathrm{~m}$, ta cần thu dữ liệu từ $1 \mathrm{~h} 30$ trở lên. $O$

\section{TÀl LIỆ THAM KHẢO}

[1]. Saastamoinen, (1972), "Atmospheric correction for the troposphere and stratophere in radio ranging of satellites", in the Use of Artificial Satellites for Geodesy, Geophysics Monograph, 15, AGU, Wasington D.C.

[2]. A. Niell, (1996), "Global mapping functions for the atmosphere delay at radio wavelengths", Journal of Geophysical Research, 101(B2), 3227-3246.

[3]. Alfred Kleusberg and P.J.G. Teunissen, (1996), "GPS for Geodesy", Springer-Verlag, Berlin.

[4]. David Wells, N. Beck, ..., (1999), "Guide to GPS Positioning", Department of Geodesy and Geomatics Engineering, University of New Brunswick, Canada.

[5]. Witchayangkoon B., (2000). "Elements of GPS Precise Point Positioning" Doctoral dissertation, Department of Spatial Information Science and Engineering, Graduate School, University of Maine, 265.

[6]. Gao Y. and Shen X., (2002), "A New Method for Carrier-Phase-Based Precise Point Positioning" Navigation, 49(2), 109-116.

[7]. Beran T., D. Kim, and R.B. Langley, (2003), "High-Precision Single-Frequency GPS Point positioning", Proceedings of the 16th International Technical Meeting of the Satellite Division of The Institute of Navigation, Portland, Oregon, U.S.A., 9-12 September 2003; The Institute of Navigation, Alexandria, Virginia, U.S.A.; pp. 1192-1200.

[8]. Alfred Leick, (2004), "GPS Satellite Surveying", John Wiley and Sons, Canada.

[9]. Beran, T., S. Bisnath, R.B. Langley and L. Serrano, (2005), "High-Accuracy Point Positioning with Low-Cost GPS Receivers: How Good Can It Get?", Proceedings of ION GPS/GNSS 2005, 18th international Technical Meeting of the Satellite Division of The Institute of Navigation, Long Beach, CA, 13-16 September 2005, The Institute of Navigation, Alexandria, Virginia, U.S.A., pp. 1524-1534.

[10]. Chalermchon Satirapod and Somchai Kriengkraiwasin, (2006), "Performance of Single-frequency GPS Precise Point Positioning", 5th Annual International Conference and Exhibition on Geographical Information Technology and Àpplications "Map Asia 2006", 29/08/2006 - 01/09/2006 Queen Sirikit National Convention Center, Bangkok, Thailan.

[11]. Nguyễn Ngọc Lâu, (2007), "Định vị điểm đơn bằng GPS hiện nay có thể đạt độ chính xác bao nhiêu?", Hội nghị Khoa học và Công nghệ lần thứ 10, Đại học Bách Khoa TP HCM.

(Xem tiếp trang 80) 
nghiệm, chuyển giao công nghệ nhằm nâng cao uy tín và thương hiệu của Viện.

Tuy nhiên vẫn còn một số tồn tại cần khắc phục như: Việc thực hiện đề tài nghiên cứu khoa học của một số chủ nhiệm đề tài vẫn còn chậm so với tiến độ. Công tác thanh quyết toán các đề tài, dự án còn chậm, một số đề tài, dự án chưa đảm bảo nội dung, trang thiết bị, kinh phí phục vụ nghiên cứu khoa học còn hạn chế.

Sang năm 2011 Viện cố gắng phát huy những thành tích đã đạt được, khắc phục những nhược điểm còn tồn tại để hoàn thành xuất sắc những nhiệm vụ Bộ giao.

Phát biểu chỉ đạo tại Hội nghị, ông Nguyễn Mạnh Hiển, Thứ trưởng Bộ Tài nguyên và Môi trường đã khen ngợi những thành tích mà Viện Khoa học Đo đạc và Bản đồ đã đạt được trong năm 2010, đặc biệt là hoàn thành các đề tài nghiên cứu khoa học đúng thời hạn, chúc Viện sang năm 2011 cố gắng hơn nữa để đạt được nhiều thành tích xuất sắc hơn. Tuy nhiên Viện cần mở rộng hợp tác khoa học hơn nữa với các đơn vị bạn trong Bộ cũng như ngoài Bộ và hợp tác quốc tế để đưa thương hiệu Viện lên tầm cao mới.

Ông Hà Minh Hòa, viện trưởng Viện Khoa học Đo đạc và Bản đồ thay mặt cán bộ, viên chức, những người lao động Viện cám ơn những lời chúc tốt đẹp của thứ trưởng Nguyễn Mạnh Hiển và hứa toàn Viện sang năm 2011 sẽ cố gắng hơn nữa để hoàn thành xuất sắc những nhiệm vụ được giao. $O$

\section{Đ!̣NH V!̣ ĐIỂM CHÍNH XÁC.....}

(Tiếp theo trang 34)

[12]. Đinh Viết Chủng, Nguyễn Ngọc Lâu và Lê Văn Trung, (2007), "Úng dụng kỹ thuật Neural Network sửa chữa trượt chu kỳ trong trị đo pha GPS", Proceedings of the 10th conference on science and technology, pp. 73-79.

[13]. Nguyễn Ngọc Lâu, (2007), "Khai thác các máy định vị cầm tay GPS trong Trắc địa", Tạp chí Địa Chính, V. 1, pp. 3-7.

[14]. Beran, T., (2008), "single-frequency, single-receiver terrestrial and spaceborne point positioning", Technical report no. 257, Department of Geodesy and Geomatics Engineering, University of New Brunswick, Fredericton, Canada, 185 pp.O

\section{SUMMARY}

PRECISE POINT POSITIONING WITH LOW-COST GPS RECEIVERS

\section{Dr. Nguyen Ngoc Lau}

\section{Geodetic subject, Ho Chi Minh city polytechnic university}

We collect data from some Garrmin hand held receivers and process them by two different methods i) correct ionospheric delay using IGS ionospheric grid and ii) estimate ionospheric zenith delay and gradient components. The processing results show that positioning accuracy is as higher as processing longer length data. With data length from $5 \mathrm{~min}-$ utes to more than 300 minutes, the horizontal accuracy is $1.50-0.15 \mathrm{~m}$ and vertical is 2.70 $-0.60 \mathrm{~m}$. Method ii) provides better accuracy than method i) about $0.2 \mathrm{~m}$ and $0.8 \mathrm{~m}$ in horizontal and vertical components respectively. $O$ 